\title{
Engineering $\mathrm{Si}$ doping in anatase and rutile $\mathrm{TiO}_{2}$ with oxygen vacancy for efficient optical application
}

\author{
S. Koley ${ }^{1}$ \\ 1 Department of Physics, North Eastern Hill University, Shillong, Meghalaya, 793022 India
}

\begin{abstract}
In this paper, I demonstrate a density functional theory plus dynamical mean field theory study on the electronic properties of doped $\mathrm{TiO}_{2}$ rutile as well as another tetragonal phase anatase with oxygen vacancy. The density of states and optical properties have been obtained from the electronic structure applying screened hybrid exchange correlation density functionals. All the single-particle excitations are treated within the dynamical mean field theory for independent quasiparticles. For optical properties, excitations are considered by solving the Bethe-Salpeter equation for Coulomb correlated electron-hole duo. On this theoretical basis, band structure and optical spectra for the two structures of $\mathrm{TiO}_{2}$ are provided. Further, I compared the present results with earlier optical data of parent structure and established the increased optical efficiency in doped $\mathrm{TiO}_{2}$ with oxygen vacancy in both the structure.
\end{abstract}

Nanoparticles exhibit surprising electronic and magnetic properties and thus they are having a number of applications in the industrial sectors like opto-electronics, bio-medical, cosmetics and many others $\stackrel{1}{-}$. The properties like hardness, rigidity, surface to volume ratio distinguish nanoparticles from its bulk systems. $\mathrm{TiO}_{2}$ is one of the most renowned materials among the oxides for its variety of industrial applications. The transition metal-oxygen bond here is having a property of increasing co-valency with oxygen. Its catalytic property is used in photocatalysis for organic synthesis. Moreover $\mathrm{TiO}_{2}$ is largely used in white painting, sensors, photovoltaic devices, food preservatives or coloring, cosmetics and in cancer treatment $\underline{\underline{2}-\underline{\underline{5}}}$.

The $\mathrm{TiO}_{2}$ polymorphs are predominantly three crystal types, rutile (tetragonal $(4 / \mathrm{mmm})$, space group: $P 4_{2} / m n m D_{4 h}^{14}$ ), brookite (orthorhombic $\left.(\mathrm{mmm}), \mathrm{Pbca} D_{2 h}^{15}\right)$ and anatase (tetragonal $(4 / \mathrm{mmm})$, $\left.I 4_{1} / a m d D_{4 h}^{19}\right)$. Among them only rutile and anatase have a major role in industry based applications. Experimental results on $\mathrm{TiO}_{2}$ brookite is little short due to its tough preparations and rare appearance $\underline{\underline{6}}$. The nanostructure of this transition metal oxide material affects the phase behavior and the thermal stability of the material. Moreover it also has influence in industrial applications. Nanostructure of this TMO shows band gap changes as inverse square of particle size $e^{7}$. The electrical conductivity is also affected due to the grain boundaries. Since it is a non-toxic semiconductor material with cost effective availability, and long term stability $\mathrm{TiO}_{2}$ has been taken into account for photovoltaic applications. The optical gaps are reported to be slightly above $3 \mathrm{eV}$ for all the three polymorphs (rutile: $3.0 \mathrm{eV}$, anatase: $3.4 \mathrm{eV}$ and brookite: $3.3 \mathrm{eV}), \underline{\underline{8}} \underline{\underline{-10}}$ so $\mathrm{TiO}_{2}$ shows photoactivity in the UV region and acts as inefficient active solar cell. Though in dye-sensitized solar cells it acts as an economically viable and good photoactive dye $\underline{\underline{11}}-\underline{13}$.

Since $\mathrm{TiO}_{2}$ is an important semiconductor having industrial applications with large band-gap and frequently used in daily life, enormous research has been already reported 14 . Also $\mathrm{TiO}_{2}$ has good applications for photocatalytic reactions, including earth abundance and resistance to photocorrosion $\frac{15}{2}$. But there is a great disadvantage of using $\mathrm{TiO}_{2}$ owing to its low quantum effficiency in photocatalytic reactions and high recombination of photogenerated electron-hole pairs. So a lot of methods have been used to modify the properties of $\mathrm{TiO}_{2}$ so that the lifetime of photogenerated electron-hole pairs increase and reduce the band gap. Among them doping $\mathrm{TiO}_{2} \frac{16,17}{1}$, co-doping with two or more ions ${ }^{15,18}$, hybridization with carbon materials $\frac{19-21}{}$, coupling with small band gap semiconductor ${ }^{22.23}$ are known to make metal core $\mathrm{TiO}_{2}$ shell composite photocatalysts. Recently defect induced changes in light absorption properties are also reported $\underline{24.25}$.

Among all other defects found in $\mathrm{TiO}_{2}$, oxygen vacancies are very important and appear in many metal oxides$^{26}$. Oxygen vacancies are easy to manipulate in metal oxides and can change the properties of oxides drastically. Both theoretical calculations and experimental characterizations $\frac{27-29}{2}$ prove that oxygen vacancies in $\mathrm{TiO}_{2}$ are essential for photocatalysis and can lead to ferromagnetism and act as a magnetic semiconductor in spintronics. In principle, the oxygen vacancies in $\mathrm{TiO}_{2}$ lead to the formation of unpaired electrons or $\mathrm{Ti}^{3+}$ centers, which also make donor levels in the band structure of $\mathrm{TiO}_{2}$. Additionally, oxygen vacancies are believed to affect the electron-hole recombination process in photocatalysts, causing a change in chemical rates that depends on charge transfer from either electrons or holes. Several investigations also reported that oxygen vacancy result in metallic conductivity $\stackrel{30}{\underline{\underline{n}}}$ at room temerature but resistivity increases with cooling.

For all the above reasons, still there is a growing interest in the development of oxygen vacancy and doping in $\mathrm{TiO}_{2}$ and thereby exploring the properties for orbital composition and magnetic moment. In this article I will focus on these for the defect induced $\mathrm{TiO}_{2}$ polymorphs rutile and anatase from theory using $\mathrm{Si}$ as dopant. I will employ a combination of density functional theory(DFT) and dynamical mean field theory(DMFT) and find out 
(a)

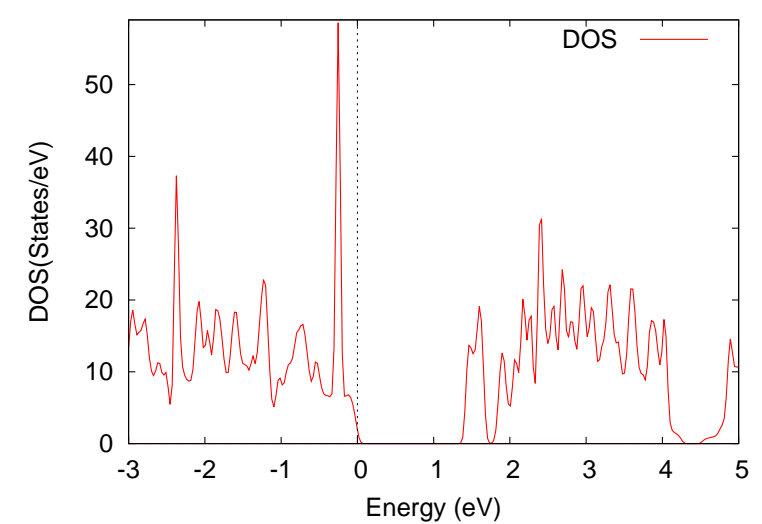

(b)

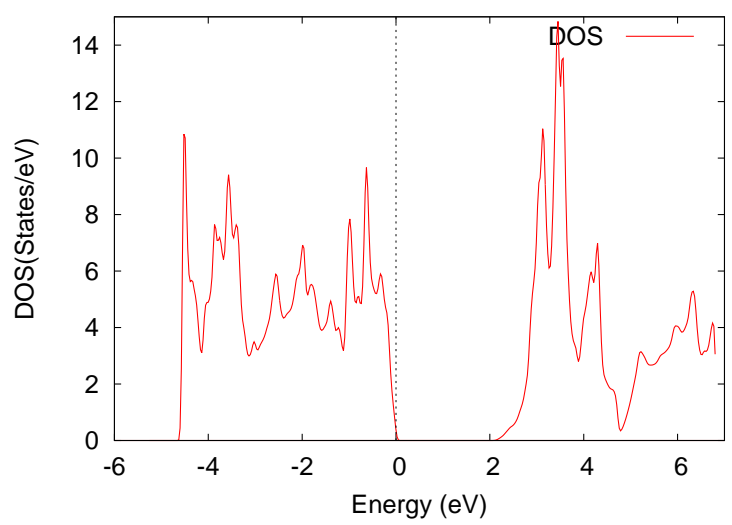

FIG. 1. (Color Online) Density of states for pristine (a)rutile $\mathrm{TiO}_{2}$ and (b)anatase $\mathrm{TiO}_{2}$. Total density of states unveils the band gap in both the cases.

optical constants to show increased efficiency.

\section{METHODOLOGY}

The structures of the three main $\mathrm{TiO}_{2}$ polymorphs are well portrayed by the two complementary $T i_{x} O_{y}$ building block. Rutile and brookite consists of corner and edgesharing $\mathrm{TiO}_{6}$ units. In rutile, the band gap is about $3.1 \mathrm{eV}$ and in ionic representation each $\mathrm{Ti}$ atom shares four electron with two oxygen atoms resulting in $T i^{4+}$ and $O^{2-}$. The valance band and conduction band in this wide band-gap semiconductor are mainly derived from $O-2 p$ and $T i-3 d$ orbitals respectively. So when a oxygen vacancy is introduced two electrons are donated to the system.

The defect induced supercell structures of rutile$\mathrm{TiO}_{2-\delta}$ are relaxed on the $\mathrm{DFT}^{32}$ level within the generalized-gradient approximation using the PerdewBurke-Ernzerhof (GGA-PBE) functional in the WIEN2K code ${ }^{31}$. The muffin-tin radii, $R_{M T}$ are chosen as 2.0 a.u. for $\mathrm{Ti}$, and 1.6 a.u. for $\mathrm{O}$. The parameter, $R k_{\max }\left(R k_{\max }\right.$ stands for the product of the smallest atomic sphere radius $R_{M T}$ times the largest k- vector $k_{\max }$ ) is chosen to be 7.0 and $2000 \mathrm{k}$-points with a $10 \times 10 \times 20 \mathrm{k}$-mesh is used here for structural optimization. Further, all atoms are structurally relaxed until the maximum force is smaller than $0.01 \mathrm{eV} / \AA$. The self consistent field (scf) computations are conducted till an energy accuracy of $0.001 \mathrm{eV}$ is achieved. The theoretical lattice constants obtained from our calculation are in agreement with previous results of parent $\mathrm{TiO}_{2}$. Then the band structure and the atom-resolved density of states are calculated from the converged scf calculations.

Here I have used charge self-consistent DFT+DMFT framework which is based on the pseudopotential approach for the DFT part and the continuous-time quantum-Monte-Carlo method, as implemented in the embedded dynamical mean field theory (EDMFTF) package ${ }^{33}$, for solving the DMFT impurity problem. I used the GGA+PBE functional form within the KohnSham cycle. EDMFTF package implements a combined DFT and DMFT derived from the stationary Luttinger-Ward functional. In this package the exact double-counting of DFT and DMFT, Coulomb interaction and SOC are accomplished nicely and the Greens function is determined self-consistently. DFT+DMFT treatment has been successful in explaining theories of strongly correlated electron systems ${ }^{34}-39$. In the theoretical model, the non-interacting Hamiltonian is added

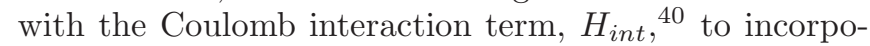
rate the effects of correlated Ti-3d orbitals. A self energy functional $\left(\Sigma_{d c}\right)$ is added to take care of the double counting. Thus the total Hamiltonian, except the $\Sigma_{d c}$ term is expressed as,

$$
H=\sum_{k, a, \sigma} \epsilon_{k, a} c_{k, a, \sigma}^{\dagger} c_{k, a, \sigma}+U \sum_{i, a} n_{i a \uparrow} n_{i a \downarrow}+
$$

$$
U^{\prime} \sum_{i, a, b, \sigma, \sigma^{\prime}} n_{i a \sigma} n_{i b \sigma^{\prime}}
$$

where $\epsilon_{k, a}$ is the band dispersion and $U$ and $U^{\prime}$ are the intra- and inter-orbital Coulomb interaction terms between electrons with opposite spins of a orbital and between electrons with same spins in two orbitals respectively. The coulomb interaction terms are varied over a realistic range to get nice agreement with earlier experiments on $\mathrm{TiO}_{2}$ and the total Hamiltonian is solved using the DMFT method. The correlated orbitals are then handled dynamically within the DMFT based on orbital projection-embedding scheme achieved via the EDMFT package. The impurity solver used in this DMFT code is the continuous time quantum Monte Carlo(CT-QMC) in the hybridization expansion method $\stackrel{41}{ }$. The parameters, namely, the Coulomb interaction $U$, and the inverse temperature, $\beta\left(=1 / k_{B} T\right)$ are varied within an experimentally realizable range to get the behavior of the oxygen vacancy in the system. The DFT+DMFT calculations are converged with respect to the charge density, chemical potential and the self energy. Finally the maximum entropy method $\frac{42}{2}$ is used for the analytical continuation 
(a)

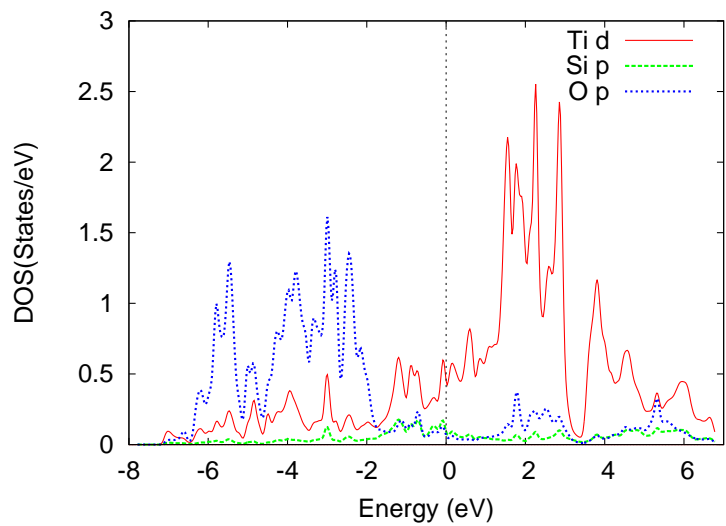

(b)

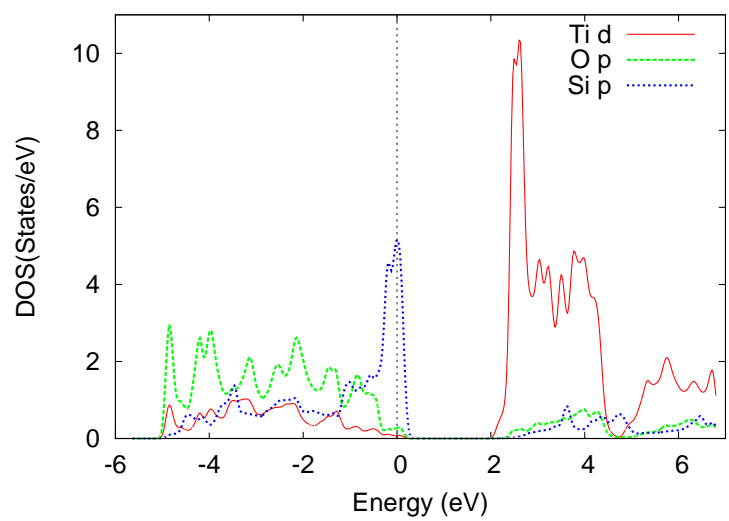

FIG. 2. (Color Online) Density of states for doped (a)rutile $\mathrm{TiO}_{2}$ and (b)anatase $\mathrm{TiO}_{2}$. Doping is induced by Si to engineer oxygen vacancy in $\mathrm{TiO}_{2}$. Density of states plots reflects the band gap is closed in both the cases and Fermi level has finite weight of impurity levels and Ti-d band.

of the self-energy from the imaginary axis to real frequencies with an auxiliary Greens function. Then from the real frequency Greens function, the momentum resolved spectral functions and the density of states are obtained.

\section{RESULTS}

The choice of coulomb interactions $\left(U\right.$ and $\left.U^{\prime}\right)$ has an important role for getting the electronic properties of the doped transition metal oxide with vacancy. For rutile and anatase $\mathrm{TiO}_{2}$ I have chosen to calculate for a reasonable range of $U$ values and found that for $U=8.0$ $\mathrm{eV}$ and $U^{\prime}=6.8 \mathrm{eV}$ the band gap energies exactly agrees well with the experimental results -10 . The orbital dependent density of states (DOS) for pristine rutile and anatase $\mathrm{TiO}_{2}$ is shown in Fig.1. Though theoretical and experimental band gap have slight difference but that should not affect the electronic structure and transport properties of defect induced $\mathrm{TiO}_{2}$ polymorphs. To create the outcome of oxygen deficiency a realistic model with one oxygen atom eliminated from the $4 \times 4 \times 6$ supercell of anatase and rutile $\mathrm{TiO}_{2}$ is formed. In Fig.2 the orbital (a)

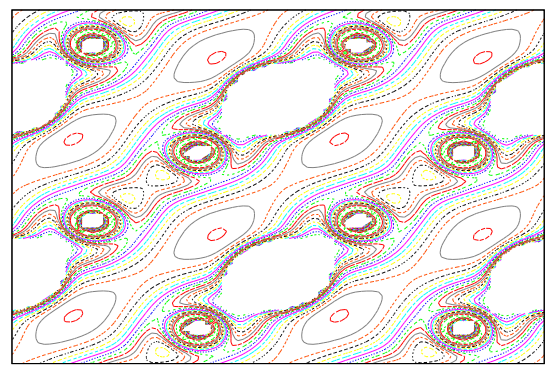

(b)

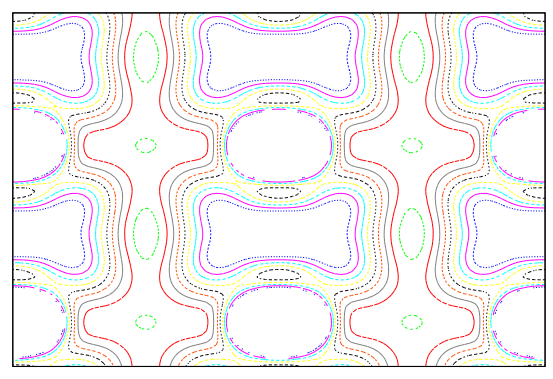

FIG. 3. (Color Online) (a) Electronic charge density plot for (a) rutile and (b) anatase phase of $\mathrm{TiO}_{2}$ in the plane containing $\mathrm{Ti}, \mathrm{O}, \mathrm{Si}$. Charge density plots shows more conducting region in comparison to parent $\mathrm{TiO}_{2}$.

dependent DOS for the doped rutile and anatase $\mathrm{TiO}_{2}$ with oxygen vacancy are displayed. Each $\mathrm{TiO}_{2}$ is having oxygen atoms and adjacent $\mathrm{Ti}$ atoms. In rutile structure among $\mathrm{Ti}$ atoms two are symmetrically equal so Ti-O bond length is somewhat different. Removal of an oxygen atom effects in three $\mathrm{Ti}$ bonds (with $\mathrm{d}$ character) pointing in the direction of the oxygen vacancy position. However the vacancy position is occupied by two electrons. The resultant orbital density of states show impurity states predominantly emerge from the $T i-d$ orbitals and doped atoms ( $S i$ is used here) and new states develop in the band gap. The symmetrically equivalent $T i$ atoms will have same DOS (not shown here). The partial charge density distribution is also shown in Fig.3 corresponding to the defect states in both rutile and anatase $\mathrm{TiO}_{2}$. The partial charge density distribution shows that vacancy of one oxygen atom in both the $\mathrm{TiO}_{2}$ lattice develops a highly conducting region in real space, and the Ti atoms that were primarily having bond with the oxygen become localized in the vacanct position. Due to the impurity doping new energy levels come into the band gap which indicates that compared with both the pristine $\mathrm{TiO}_{2}$, there is extra charge on the $\mathrm{O}$-deficient $\mathrm{Ti}$ ions in the presence of an oxygen vacancy. As an out- 
(a)

(b)
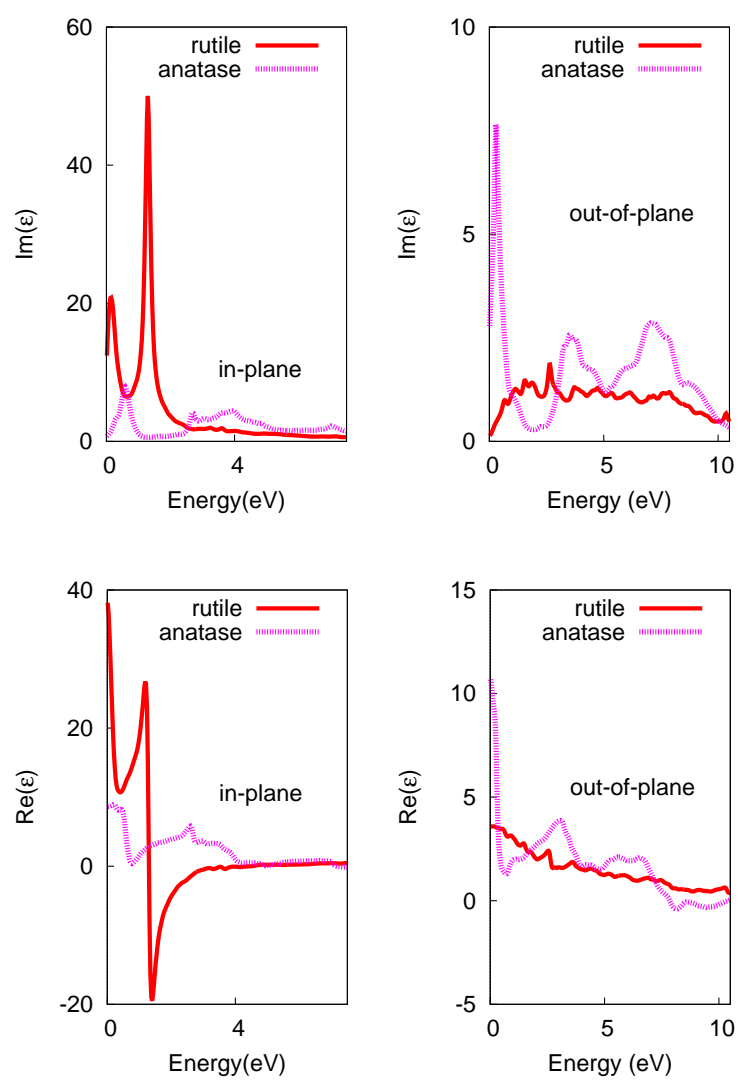

FIG. 4. (Color Online) The (a) imaginary and (b) real part of the dielectric function as calculated from DMFT for rutile and $\mathrm{TiO}_{2}$ along in-plane and out-of-plane direction The static value of real part of dielectric function has increased due to oxygen vacancy and absorption peak is also starting from zero energy making it more efficient for solar cell application.

come of doping the O-deficient $\mathrm{Ti}$ ions transform from higher valence state to a lower valence state after creating a neutral oxygen vacancy. Now since anatase $\mathrm{TiO}_{2}$ has wide applications in the field of optoelectronic devices and solar cell conversion efficiency so next I will show optical properties of oxygen deficient $\mathrm{TiO}_{2}$. The imaginary part of the dielectric function is defined as optical absorption. $\mathrm{TiO}_{2}$ has tetragonal structure and literature shows it exhibits optical anisotropy. Hence, the presence of anisotropy is also obvious for the doped systems, and it is more pronounced in the low energy part of the spectra. So I present optical properties in both the in-plane and out-of plane direction.

The absorption spectra (Fig.S1(Supplementary)) of undoped rutile structure shows $3.0 \mathrm{eV}$ as initial energy for absorption from DMFT calculations while dielectric function at zero energy is having constant value of 5.75 and 5.55 along in-plane and out-of-plane direction which is consistent with earlier results on rutile $\mathrm{TiO}_{2} \underline{43}, 44$. So our calculation with DFT+DMFT spectra shows very good agreement with earlier results and the precise absorption spectra from oxygen vacant states also can be achieved. Similarly for anatase structure the onset of absorption starts from $2.4 \mathrm{eV}$ and real part of dielectric function is having values 5.35 and 5.2. Now I will show optical absorption spectra which carry a number of important properties like refractive index $(\eta)$, relectivity $(\mathrm{R})$, optical conductivity $(\sigma)$, extinction coefficient $(\kappa)$ and optical loss spectra. In DFT+DMFT from EDMFTF package all of these can be calculated. The theoretically calculated in-plane and out-of-plane refractive indices for rutile $\mathrm{TiO}_{2}$ are in close agreement with the experimental results 44 . Therefore this DFT+DMFT method is good enough to produce the doped $\mathrm{TiO}_{2}$ case.

The many body theory in rutile $\mathrm{TiO}_{2}$ is recently reported $\frac{45}{5}$ but for anatase structure it is still not reported. DMFT can unveil the presence of defects which affects the optical properties of pristine $\mathrm{TiO}_{2}$ structures. Fig.4a and Fig.4b shows real and imaginary part of dielectric function of doped rutile and anatase $\mathrm{TiO}_{2}$. In both the case though the nature of the absorption pattern is same but in pristine $\mathrm{TiO}_{2}$ there was no absorption peak below $3 \mathrm{eV}$ but in doped cases it is different and absorption peaks start from almost zero energy. However like parent structure optical anisotropy is present in the doped system also and it is pronounced in both the structure. Though presence of low energy peaks in both the case reflect the two directions as optically active for polarization of light. The reason for optical activity can be explained from the band structure itself. Since oxygen vacancy is created and the system is doped with $\mathrm{Si}$, which is having a tendency to accept negative charges so the Fermi level will be changed towards conduction band minima. So here Si will act as n-type dopant and $p_{x}, p_{y}$ and $p_{z}$ orbitals actively participate in optical transitions at low energy. Thus n-type dopants make rutile and anatase $\mathrm{TiO}_{2}$ optically active in low energy for light polarized along $x y$ and $z$ direction.

Now Fig. 5 shows optical constants in in-plane and outof-plane for the enrgy range $10 \mathrm{eV}$ which is reasonable since I have taken DFT bands also in this range. The refractive index is having a peak (Fig.4d) in the infrared and visible range followed by a fast decrease in the ultraviolet range for rutile structure when electric field is in-plane while there is a slow decrease for out-of plane direction. For anatase structure refractive index follow opposite behaviour, with two small peaks in in-plane visible and infrared range and a slow decrease in the UV range while out-of plane refractive index is having a large peak in infrared region and decreasing fast in the UV region. Depending on the structure refractive index is near about 2 in the visible range of the electromagnetic spectrum. A similar behaviour is found for extinction coefficient (Fig.4b), which is responsible for attenuation of electric field and is a cause of dielectric loss. Next the dielectric loss is presented in Fig.4f which shows highest loss in low wavelength region. According to all these results optical constants can be changed by oxygen vacancy or doping. Next I have theoretical optical conductivity also from DMFT. Optical conductivity in turn 
(a)

(d)
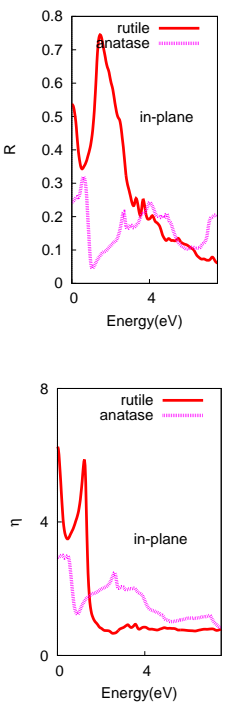

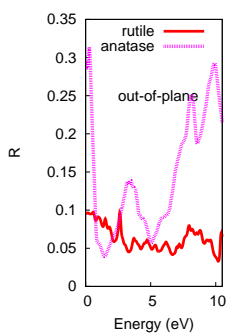

(b)

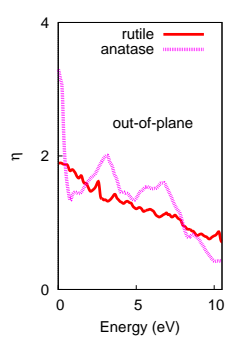

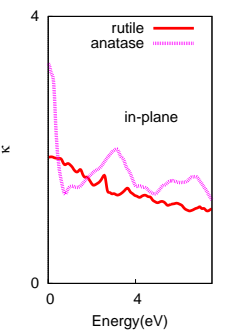

(e)

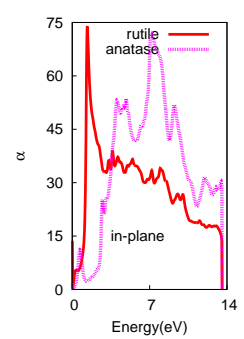

(c)
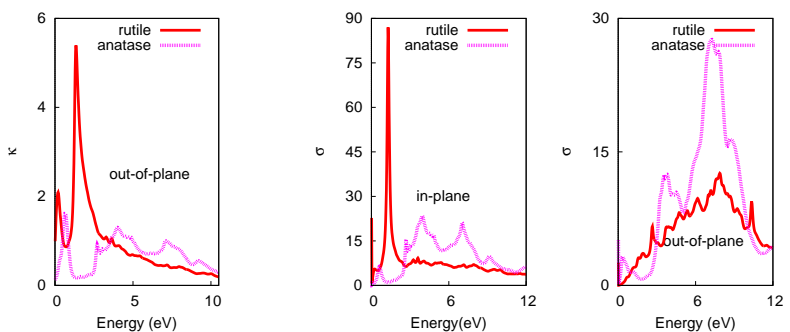

(f)

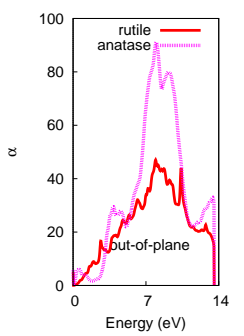

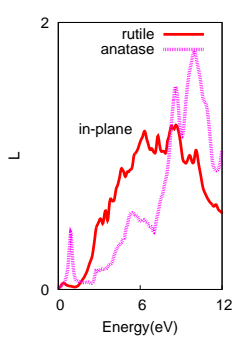

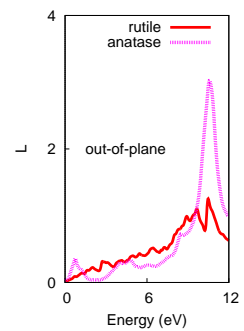

FIG. 5. (Color Online) The DMFT optical constants plot: (a)reflectivity, (b)extinction coefficient, (c) optical conductivity, (d)refractive index, (e)absorption coefficient and (f) dielctric loss spectra for rutile and anatase $\mathrm{TiO}_{2}$ crystal structure. Optical constants are plotted for in-plane and out-of-plane direction.

is related to refractive index (Fig.4d), absorption coefficient (Fig.4e) and light speed. Optical conductivity is the electrical conductivity as a result of movement of the charge carriers due to alternating electric field generated by electromagnetic wave. It is observed that optical conductivity increases in doped $\mathrm{TiO}_{2}$ due to the new energy levels in the bad gap. The impurity levels in the band gap facilitate the electrons from the valance band transmits to the conduction band.

In conclusion, the electronic structure of rutile and anatase $\mathrm{TiO}_{2}$ has been investigated using DFT+DMFT method with the introduction of oxygen vacancies and $\mathrm{Si}$ doping. Electronic charge density shows formation of a high conducting region in both $\mathrm{TiO}_{2}$. The theoretical results are consistent with earlier findings of n-type dopant in $\mathrm{TiO}_{2}{ }^{46}$. Optical excitations are found to be modi- fied by oxygen vacancy while they remain anisotropic as in the parent system. Optical activity in both in-plane and out-of-plane direction is found for doped rutile and anatase structure. As a result of defect and doping optically allowed transitions are from very low energy which improves its applicability in opto electronic and solar absorption properies.

\section{ACKNOWLEDGEMENT}

S. Koley acknowledges DST women scientist grant SR/WOS-A/PM-80/2016(G) for finance and also thank Prof. M C Mahato for mentoring and useful conversations.
1 E. Roduner, Chem. Soc. Rev. 35, 583 (2006); A.M. Smith and S. Nie, Acc Chem Res. 43, 190 (2010).

2 M.R. Hoffman, et al., Chem. Rev. 95, 69 (1995).

3 A. Maldoti, et al., Chem. Rev. 102, 3811 (2002).

4 P. Bonhole, Thin Solid Films 350, 269 (1999).

5 L.G. Phillips, et al., J. Dairy Sci. 80, 2726 (1997).

6 D. Dambournet, I. Belharouak and K. Amine Chem. Mater. 22, 1173 (2009).

7 V. Luca, et al., J. Phys. Chem. B 102, 10650 (1998).

8 J. Pascual, J. Camassel and H. Mathieu Phys. Rev. B 18, 6842 (1978).

9 H. Tang, F. Lévy, H. Berger and P.E. Schmid Phys. Rev. $B$ 52, 7771 (1995).

10 A. Mattsson and L. Österlund J. Phys. Chem. C 114, 14121 (2010).
11 U. Bach et al., Nature 395, 583 (1998).

12 M. Grätzel., MRS Bull 30, 23 (2005).

13 A. Hagfeldt et al., Chem. Rev. 110, 6595 (2010).

14 X. Pan et al., Nanoscale 5, 3601 (2013).

15 X. Chen and S. S. Mao, Chem. Rev. 107, 2891 (2007).

16 W. Choi, A. Termin and M. R. Hoffmann, J. Phys. Chem. 98, 13669 (1994).

17 Z.-R. Tang, Y. Zhang and Y.-J. Xu, ACS Appl. Mater. Interfaces 4, 1512i (2012).

18 D. Li et al., Environ. Sci. Technol. 42, 2130 (2008).

19 Y. Zhang, Z.-R. Tang, X. Fu and Y.-J. Xu, ACS Nano 5, 7426 (2011).

20 N. Zhang, Y. Zhang, X. Pan, M.-Q. Yang and Y.-J. Xu, J. Phys. Chem. C 116, 18023 (2012). 
21 Y.-J. Xu, Y. Zhuang and X. Fu, J. Phys. Chem. C 114, 2669 (2010).

${ }^{22}$ G.-S. Li, D.-Q. Zhang and J. C. Yu, Environ. Sci. Technol. 43, 7079 (2009).

23 S. Liu, N. Zhang, Z.-R. Tang and Y.-J. Xu, ACS Appl. Mater. Interfaces 4, 6378 (2012).

24 J. Nowotny, T. Bak, M. K. Nowotny and L. R. Sheppard, Int. J. Hydrogen Energy 32, 2630 (2007).

25 M. K. Nowotny, L. R. Sheppard, T. Bak and J. Nowotny, J. Phys. Chem. C 112, 5275 (2008).

26 G. Pacchioni, ChemPhysChem 4, 1041 (2003).

27 I. Nakamura et al.,J. Mol. Catal. A: Chem. 161, 205 (2000).

28 T. Thompson and J. Yates, Top. Catal. 35, 197 (2005).

29 Z. Zhang et al., J. Am. Chem. Soc. 128, 4198 (2006).

30 T. Gu, J. Appl. Phys. 113, 033707 (2013).

31 P. Blaha, K. Schwarz, G.K.H. Madsen, D. Kvasnicka, and J. Luitz, WIEN2k, An Augmented Plane Wave + Local Orbitals Program for Calculating Crystal Properties (Wien:Karlheinz Schwarz, Techn. Universitat Wien, 2001).

32 T.L. Loucks, Augmented Plane Wave Method (New York:Benjamin, 1967); O.K. Andersen, Solid State Commun. 13, 133 (1973); E. Wimmer, et al., Phys. Rev. B 24, 864 (1981).

${ }^{33}$ K. Haule, C. Yee, and K. Kim, Phys. Rev. B 81, 195107 (2010).

34 K. kim et al., Nat. Mater. 17, 794 (2018).

35 A. Eugene et al., Science 359, 186 (2018).

36 A. Taraphder, S. Koley, N.S. Vidhyadhiraja, and M.S. Laad, Phys. Rev. Lett., 106, 236405 (2011).

37 S. Koley, M.S. Laad, N.S. Vidhyadhiraja, and A. Taraphder, Phys. Rev. B, 90, 115146 (2014).

38 A. Georges et al., Rev. Mod. Phys. 68, 13 (1996).

39 S. Koley, Solid State Commun. 251, 23 (2017).

40 S. Koley, and S. Basu, arXiv:1904.03698.

41 K. Haule, Phys. Rev. B 75, 155113 (2007).

42 M. Jarrell and J. E. Gubernatis, Phys. Rep. 269, 133 (1996).

43 S. Wemple, The Journal of Chemical Physics 67, 2151 (1977).

44 R. Gonzalez, R. Zallen, and H. Berger, Phys. Rev B 55, 7014 (1997).

45 M. O. Atambo et al., Phys. Rev Materials 3045401 (2019).

46 P. Basera, S. Saini, and S. Bhattacharya, arXiv:1905.10569.

(a)
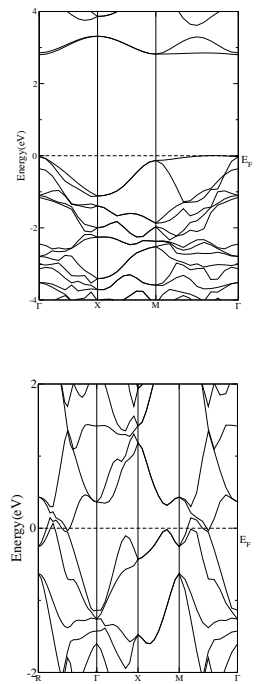

(c)

FIG. S1. (Color Online) The plots show band structure of pristine (a)rutile and (b)anatase and oxygen deficient (c) rutile and (d) anatase $\mathrm{TiO}_{2}$ structure.

\section{SUPPLEMENTARY INFORMATION}


(a)
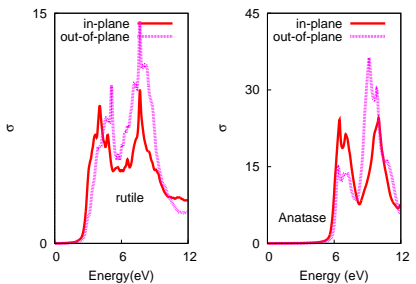

(b)
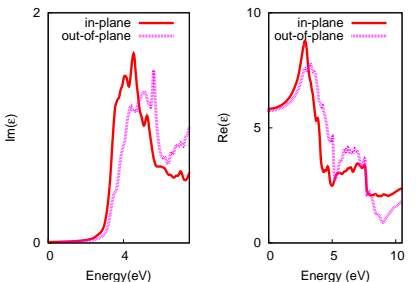

(c)
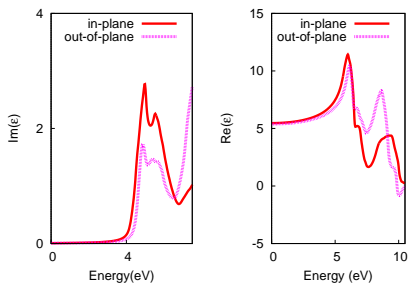

FIG. S2. (Color Online) (a) Optical Conductivity, (b) dielectric function of rutile and (c) dielectric function of anatase of parent $\mathrm{TiO}_{2}$ structure.

(a)

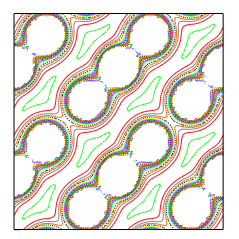

(b)

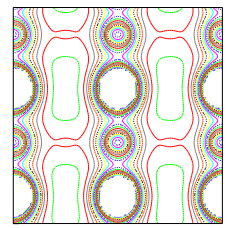

FIG. S3. (Color Online) Electronic charge density of pristine (a) rutile and (b) anatase $\mathrm{TiO}_{2}$ structure in the plane containg $T i-O$. 